\section{References}

1. Shanafelt TD, Hasan O, Dyrbye LN, et al. Changes in burnout and satisfaction with work-life balance in physicians and the general US working population between 2011 and 2014. Mayo Clin Proc 2015; 90:1600-13.
2. Canadian Medical Association. CMA National Physician Health Survey; October 2018. Available: https://www.cma.ca/sites/default/ files/pdf/News/nph-survey-e.pdf (accessed 2019 Jan. 14).

3. Touzin C. Médecins en détresse. La Presse. 2018 Dec. 11. Available: www.lapresse.ca/actualites/sante/201812/10/01-5207563-medecins -en-detresse.php (accessed 2019 Jan. 14).

\title{
Ne pas passer sous silence l'épidémie de burn-out
}

Les opinions exprimées dans cet éditorial sont celles de l'auteur et ne représentent pas nécessairement celles de l'Association médicale canadienne ou ses filiales.

epuis peu, l'épuisement professionnel des médecins est sur toutes les lèvres. Problème connu depuis des années, le burn-out sort maintenant de l'ombre. Et en soi, le nombre de cas pose une menace pour la profession médicale. Aux États-Unis, on déplore environ 400 suicides de médecins chaque année. On ignore quel en est le nombre au Canada.

La Clinique Mayo a publié les résultats de son enquête sur l'épuisement professionnel en $2016^{1}$. Ce rapport révèle que la proportion de médecins présentant au moins 1 symptôme de burn-out était passée de $46 \%$ à $54 \%$ par rapport à l'année précédente. L'Association médicale canadienne a pour sa part diffusé des données sur l'expérience canadienne au cours de la dernière année ${ }^{2}$. Un médecin sur 3 faisait état de signes de dépression. Les résidents semblaient plus à risque (95\%) à l'égard de la dépression, mais était également plus susceptibles d'en parler. Le Programme d'aide aux médecins du Québec (PAMQ) existe depuis 1990. Les nouveaux cas sont sur le point de créer une congestion au sein du programme. L'an dernier leur nombre a augmenté de $20 \%$ chez les spécialistes et de $12 \%$ chez les médecins de famille 3 .

Les motifs de consultation au PAMQ varient, mais semblent liés à plusieurs facteurs environnementaux. Les médecins se sentent coincés dans une situation sans issue qui les pousse vers la retraite ou la fermeture de leurs cabinets pour des périodes indéterminées. Ils affirment aussi que les changements apportés au système ont alourdi leur tâche et rendu leurs milieux de travail dysfonctionnels; ils n'ont de contrôle sur rien. Les plus affectés sont moins âgés que la moyenne des médecins (43 ans c. 52) $)^{2,3}$.

On a assisté au dénigrement des médecins par des porte-paroles gouvernementaux dans les médias, ainsi qu'à une rationalisation des soins par les ministères de la Santé et les administrations hospitalières. La rationalisation émane du gouvernement, mais ce sont les médecins qui en portent l'odieux. Leurs plaintes concernent les contraintes de temps, le déclin des salaires et la multiplication des tracasseries bureaucratiques imposés par l'administration, qui ne font rien pour améliorer les soins. Les enquêtes menées par les ordres professionnels sur leurs membres et les poursuites constituent d'autres importants facteurs de stress. La mise en place des dossiers médicaux électroniques n'a pas non plus facilité la prestation des soins.

Les diplômés en médecine subissent sans contredit une pression inégalée pour s'adapter à un nouveau milieu de travail, sans compter l'endettement et l'incertitude professionnelle inhérente aux restrictions budgétaires. Mais qu'est-ce qui rend cette génération de médecins plus sujette à l'épuisement professionnel? Ce pourrait être simplement une sensibilité exacerbée aux difficultés de la vie, combinée à une moindre stigmatisation de ceux qui les dénoncent. Cela pourrait signifier que l'environnement est relativement le même, mais qu'on l'évalue avec des instruments plus précis.

Les conditions de travail se sont améliorées. Les résidents ne sortent plus d'un programme de 5 ans où ils ont fait 1 garde sur 2. Les nouveaux médecins disposent de programmes de soutien et ne travaillent pas plus d'heures que leurs prédécesseurs. Par contre, les soins aux patients se sont complexifiés, les tests se sont multipliés, tout comme les comorbidités. Mais la diminution des possibilités d'emploi et des postes de résidents en spécialité alliée à la recherche d'une meilleure conciliation travail-vie personnelle ajoute un élément de stress. Malheureusement, les réclamations pour burn-out semblent avoir parfois d'étranges paramètres. 
J'ai entendu des gens se plaindre d'être trop fatigués pour faire une garde, tout en augmentant leurs listes de stages. Idéalement, comme avec toute autre invalidité, il faut des paramètres diagnostiques et thérapeutiques plus objectifs pour le burn-out. C'est un problème réel qui se doit d'être mieux défini.

À l'heure actuelle, les plans de traitement varient énormément d'un cas à l'autre, sans règle. Les protocoles thérapeutiques sont disparates alors qu'ils devraient être standardisés. Comme toute autre épidémie, nous devons reconnaître l'importance de l'épuisement professionnel chez les médecins et s'y attaquer. Par contre, si on considère les diverses composantes du problème, on conclut qu'à l'avenir, il faudra former plus de médecins pour répondre à nos besoins.

\section{Edward J. Harvey, MD}

Corédacteur en chef, fournal canadien de chirurgie
Intérêts concurrents : E.J. Harvey est médecin chef chez Greybox Solutions; il est cofondateur et directeur de l'innovation médicale de NXTSens Inc, cofondateur et médecin chef de MY01 Inc. et cofondateur et directeur de Strathera Inc. Il bénéficie du soutien institutionnel de J \& J, DePuy Synthes, Stryker et Zimmer, et il fait partie du conseil d'administration de l'Orthopedic Trauma Association et de l'Association canadienne d'orthopédie.

DOI: $10.1503 /$ cjs.000919

\section{Références}

1. Shanafelt TD, Hasan O, Dyrbye LN, et al. Changes in burnout and satisfaction with work-life balance in physicians and the general US working population between 2011 and 2014. Mayo Clin Proc 2015;90:1600-13.

2. Sondage national de l'AMC sur la santé des médecins; Octobre 2018. Accessible : https://www.cma.ca/sites/default/files/pdf/News/ nph-survey-f.pdf (consulté le 14 janvier 2019).

3. Touzin C. Médecins en détresse. La Presse. Le 11 décembre 2018. Accessible : www.lapresse.ca/actualites/sante/201812/10/01 -5207563-medecins-en-detresse.php (consulté le 14 janvier 2019).

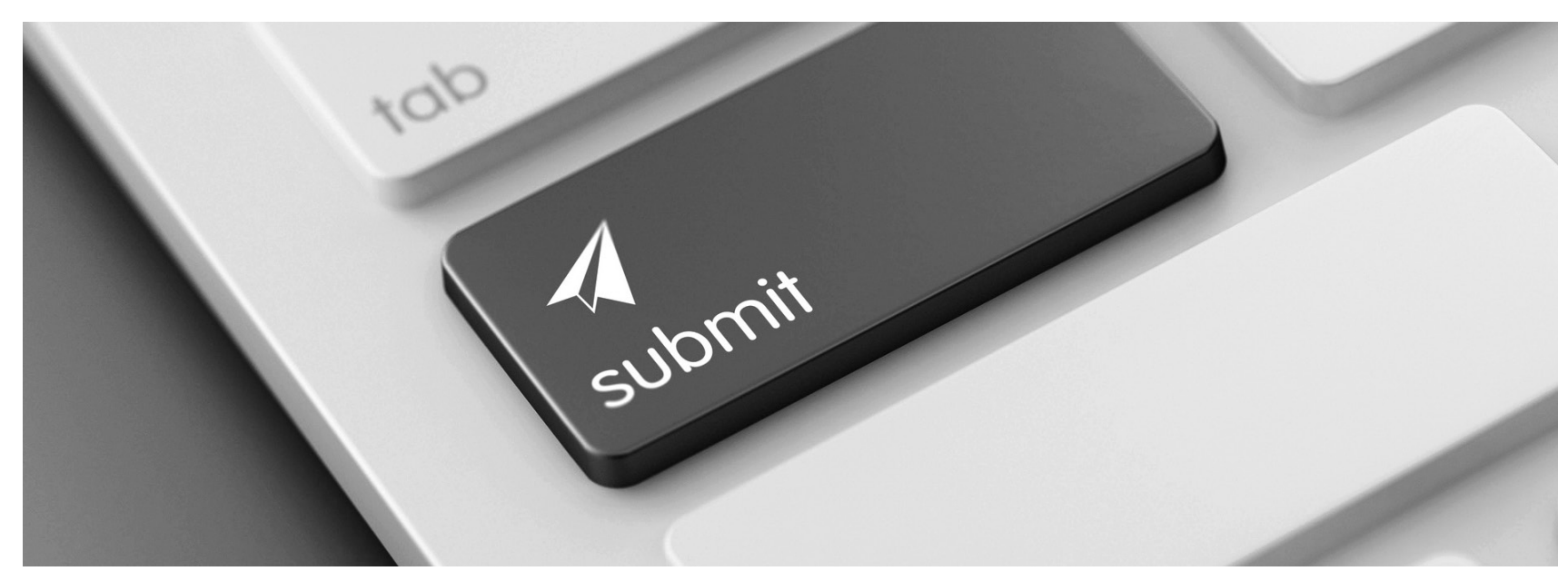

\title{
Canadian Journal

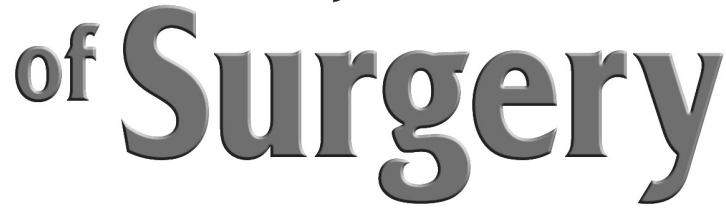

\section{Online manuscript submission and peer review}

\author{
AVAILABLE at \\ http://mc.manuscriptcentral.com/cjs
}

